\title{
Molecular characteristic of imipenem-resistant Pseudomonas aeruginosa isolated from unrinary tract infections in Southern Poland
}

\author{
M Pobiega', J Maciag², A Chmielarczyk', D Romaniszyn', M Monika Pomorska-Wesolowska', G Ziolkowski \\ J Wojkowska-Mach ${ }^{1 *}$, PB Heczko ${ }^{1}$
}

From 3rd International Conference on Prevention and Infection Control (ICPIC 2015)

Geneva, Switzerland. 16-19 June 2015

\section{Introduction}

Carbapenem-resistant Pseudomonas aeruginosa (PAR) has become a serious health problem worldwide. It is essential to understand its epidemiology as it may help to control the antibiotic resistance.

\section{Objectives}

To analyze the molecular characteristics of carbapenemresistant PAR in urinary tract infections in Southern Poland.

\section{Methods}

Antimicrobial susceptibility testing was performed. Metallo-beta-lactamases were detected. Multidrug-resistant (MDR) was non-susceptible to one antimicrobial in $\geq 3$ antimicrobial classes. Extensively-drug resistant strain (XDR) was susceptible to $\leq 2$ antimicrobial classes. MLST was performed (Curran et al,2004).

\section{Results}

The median $(\mathrm{Q} 1 ; \mathrm{Q} 3)$ age was 60 years $(54 ; 69), 33.3 \%$ were females. Among 183 urine samples contained $P$. aeruginosa, 21 imipenem-non-susceptible strains were included for further analysis. MIC50 for imipenem was $12.0 \mathrm{mg} / \mathrm{l}$. Eighteen strains (86.0\%) were resistant to meropenem $(\mathrm{MIC} 50=8.0 \mathrm{mg} / \mathrm{l})$. Sixteen strains $(76.0 \%)$ were resistant to doripenem. Based on the EDTA-assay, 9 (42.8\%) MBL-positive isolates were identified. VIM-2 was present in three isolates. No isolates with SPM nor IMP, SIM, GIM were detected. Three (14.2\%) isolates were classified as MDR, 8 as XDR (38\%). MDR/XDR strains were found more often among polimycrobial infections than monomicrobial $(\mathrm{p}=0.042, \mathrm{OR}=0.093,95 \%$ CI 0.0085-1.00). Eight XDR strains were designated to MLST typing scheme. Four strains belonged to ST235, two strains to ST 260. The remaining two strains belonged to ST654 o ST234, respectively.

\section{Conclusion}

This study indicated the emergence of MDR and XDR strains producing MBL. A high prevalence of imipenemresistant strains and MBL is a critical problem and a therapeutic challenge for clinicians. Continuous surveillance is necessary to detect the presence of MBL-producing strains. No 2012/05/N/NZ7/00786.

\section{Disclosure of interest}

None declared.

\section{Authors' details}

${ }^{1}$ Jagiellonian University Medical School, Kraków, Poland. ${ }^{2}$ Department of Dental Prophylaxis and Experimental Dentistry, Institute of Dentistry Jagiellonian University Medical College, Kraków, Poland. ${ }^{3}$ Higher School of Medicine in Sosnowiec, Sosnowiec, Poland.

Published: 16 June 2015

doi:10.1186/2047-2994-4-S1-P139

Cite this article as: Pobiega et al:: Molecular characteristic of imipenemresistant Pseudomonas aeruginosa isolated from unrinary tract infections in Southern Poland. Antimicrobial Resistance and Infection Control 2015 4(Suppl 1):P139.

TJagiellonian University Medical School, Kraków, Poland

Full list of author information is available at the end of the article 